\title{
Suicidio: el impacto del Covid-19 en la salud mental
}

\section{Suicide: the impact of Covid-19 on mental health}

\author{
Érika Benítez Camacho*
}

https://doi.org/10.36105/mye.2021v32n1.01

\section{Resumen}

El suicidio es una problemática grave de salud pública en México y en el mundo. La Organización Mundial de la Salud señala que cada año mueren por este medio aproximadamente 800 mil personas. De origen multifactorial, los factores de riesgo del suicidio incluyen elementos biológicos, psicológicos, sociales y culturales y, sobre todo, los trastornos mentales y las crisis de vida. Precisamente en estos dos ámbitos es donde se inserta el impacto que la pandemia del Covid-19 ha tenido en la población general, en quienes han dado positivo a este virus y en el personal sanitario. Pese a que aún es pronto para reconocer con seguridad la huella profunda de la pandemia en diferentes ámbitos y, en particular, en la muerte autoprovocada, es indudable que la crisis sanitaria ha develado la imperiosa necesidad de reflexionar sobre la solidaridad, el principio de sociabilidad, subsidiariedad y la vulnerabilidad del ser humano.

Palabras clave: suicidio, salud mental, trastornos mentales, principio de sociabilidad, subsidiariedad, vulnerabilidad.

\footnotetext{
* Miembro de la Asociación Psiquiátrica Mexicana y de la Academia Nacional Mexicana de Bioética. Facultad de Psicología, Universidad Anáhuac México, México. Correo electrónico: ebenitez@anahuac.mx https://orcid.org/0000-0002-8522-7145

Recepción: 20 de septiembre de 2020. Aceptación: 15 de octubre de 2020.
} 


\section{Introducción}

La muerte autoprovocada, o el acto de quitarse deliberadamente la propia vida, es un fenómeno complejo que difícilmente se decide de un momento a otro; es un proceso asociado a crisis emocionales que devienen en desesperanza, vacío existencial y pérdida del sentido de vida. Frente a la experiencia desolada de los problemas, la persona considera que la única solución a los mismos es la muerte. Los factores de riesgo y de protección para el suicidio son diversos. En su comprensión radica la posibilidad de diseñar estrategias de intervención y prevención asertivas, orientadas a la salvaguarda de lo más valioso que tiene un ser humano, su vida. Este artículo tiene por objetivo ahondar en aspectos relevantes sobre el suicidio y su vinculación con el Covid-19.

\section{El suicidio en el mundo}

El suicidio es una problemática de salud pública, que se ha incrementado significativamente en nuestro país y en el mundo. Según la Organización Mundial de la Salud (1,2), cada año se suicidan alrededor de 800 mil personas; en otras palabras, cada 40 segundos muere una persona en el mundo por esta causa. La tasa mundial de suicidio es de 10.6 por cada 100 mil habitantes (13.5 en hombres y 7.7 en mujeres); sin embargo, existen diferencias significativas dependiendo del ingreso económico de las naciones, pues, a pesar de que el $79 \%$ de las muertes autoprovocadas ocurre en los países con ingresos bajos y medianos, la tasa de suicidios es mayor en aquéllos con ingresos elevados (tasa de 10.5 vs. 11.5 respectivamente). La prevalencia de acuerdo con el sexo también se ve influenciada por la variable económica. Mientras que en los países desarrollados los hombres se suicidan casi tres veces más que las mujeres, en los países en vías de desarrollo mueren por este motivo 1.5 hombres por cada mujer (3). Estos datos permiten identificar que, en términos 
globales, los hombres se suicidan más que las mujeres. Sin embargo, existen algunas excepciones, como en China, en donde la tasa de suicidios por cada 100 mil habitantes es de 10.3 en mujeres y de 9.1 en hombres (4).

De manera general, en casi todos los países del mundo los individuos menores de 15 años se suicidan menos y las personas entre 15 y 49 años se suicidan más. Los adultos mayores de 70 años o más también tienen representatividad en este grupo (5). Desde el 2015, el suicidio es la segunda causa principal de defunción en la población mundial de entre 15 y 29 años, después de los accidentes de tránsito. En los adolescentes de entre 15 y 19 años, es la segunda causa de muerte en las mujeres (después de las afecciones maternas) y la tercera en los hombres (después de los decesos por violencia interpersonal y accidentes de tránsito) (2).

Los métodos de suicidio dependen en gran medida de factores sociales y culturales, así como del acceso que se tenga a dichos medios. No obstante, a nivel mundial el ahorcamiento es el más frecuente, seguido de las armas de fuego y de la intoxicación voluntaria con plaguicidas (este último usado mayormente en zonas rurales) $(1,3)$. Se observa, por ejemplo, que en países asiáticos como China, Taiwán, Hong Kong y Japón, el salto de una gran altura y la intoxicación con monóxido de carbono, con gas sulfuro de hidrógeno y con gas helio, son métodos suicidas comunes.

\section{La situación en México}

Si se consideran todas las regiones establecidas por la Organización Mundial de la Salud, Europa tiene la tasa de suicidios más elevada (15.4), y de ella, la región del Mediterráneo Oriental (3.9) la más baja. América tiene una tasa de 13.2 suicidios por cada 100 mil habitantes (14.8 en hombres y 11.7 en mujeres) (2).

Con una tasa de 6.1 defunciones autoprovocadas por cada 100 mil habitantes, la presencia del suicidio en América Latina es baja 
comparada con otros países en el mundo. En este rubro, México tiene una tasa de 5.1 suicidios ( 8.0 para hombres y 2.3 para mujeres). El número podría parecer bajo; sin embargo, se deben considerar dos elementos fundamentales. El primero y más importante es que toda vida es valiosa y, el segundo, que las cifras han sufrido un incremento alarmante en los últimos años. Específicamente, en los últimos 37 años ha habido un aumento de $976 \%$ en la presencia del suicidio en nuestro país (en 2017 se registraron 6,559 suicidios). Las entidades federativas con mayor tasa de suicidio en México son: Chihuahua (11.4), Yucatán (10.2), Aguascalientes (9.6), Campeche (9.1), Colima (8.5), Guanajuato (7.8), Quintana Roo (7.7), Jalisco (7.2), Baja California Sur (7.1) y Sonora (7.1) (6).

La muerte por esta causa sigue la tendencia mundial, de tal forma que es más frecuente en hombres $(80.1 \%$ de los suicidios) que en mujeres $(19.9 \%)$, lo que representa una tasa de 8.5 suicidios por cada 100 mil varones y de 2.0 por cada 100 mil féminas. En ambos casos, el principal método es el ahorcamiento, estrangulamiento o sofocación (7).

En México, el suicidio es la tercera causa de muerte en adolescentes de 15 a 19 años y la quinta en menores de 15 años (8). Seis de cada 10 muertes por suicidio se dan en personas menores a 30 años, ${ }^{1}$ mayoritariamente mujeres. ${ }^{2}$ Puntualmente, es llamativo que cuando se tiene una edad de 29 años o menos, los suicidios de las féminas representan un mayor porcentaje en nuestro país. Sin embargo, conforme aumenta la edad, el número de muertes autoprovocadas es mayor en los hombres, hasta el punto de que en personas de 65 años o más, la distribución porcentual de suicidios es de 7.7 en hombres y de 2.7 en mujeres (7).

Resulta sustancial distinguir los factores que intervienen en el comportamiento de esta variable, de manera que no sólo se desarrollen estrategias de prevención del suicidio de acuerdo con la edad y el género por separado, sino que puedan articularse maniobras de intervención, considerando la interposición de ambos ele- 
mentos, sin excluir, por supuesto, la presencia de otros factores que contribuyen a la conducta suicida.

\section{Factores de riesgo en el suicidio}

El interés por identificar las causas del suicidio ha dado pie a múltiples investigaciones que concluyen su origen multifactorial y la combinación de elementos cognitivos y neurobiológicos asociados a situaciones de vida estresantes y/o traumáticas (9).

Factores como experiencias de trauma en la infancia y/o adolescencia, disfunción familiar, comunicación familiar fracturada, violencia intrafamiliar (haberla vivido o sido testigo de ella), abuso psicológico, violencia física o sexual, acoso escolar, ausencia de un proyecto de vida, desesperanza, disminución del sentido de vida, vacío existencial y falta de una red de apoyo social, son considerados elementos de riesgo para la conducta suicida ${ }^{3}$ (10-17).

En las áreas de genética y neurobiología, los estudios han versado sobre la expresión genética y proteica implicada en el comportamiento suicida y sobre el metabolismo de la serotonina; sobre la posible identificación de genes específicos asociados a la conducta suicida, y las áreas cerebrales vinculadas con el procesamiento emocional, la percepción real o imaginaria del dolor, la ideación suicida y el intento de suicidio (18-22).

Los trastornos mentales primordialmente subyacentes a la conducta suicida son los trastornos depresivos (en particular el trastorno depresivo mayor), el trastorno de estrés postraumático, los trastornos de ansiedad (incluyendo la ansiedad generalizada, la fobia social y el trastorno de pánico), los trastornos por consumo de sustancias y los trastornos de la personalidad (23-29). Dichos trastornos se involucran con la conducta suicida no sólo por la sintomatología propia de cada trastorno o, incluso, por la comorbilidad que puede existir entre dos o más trastornos mentales y/o de la personalidad, sino también por la dificultad asociada para tolerar efec- 
tivamente el estrés, la frustración o las situaciones adversas, así como para implementar estrategias efectivas de habilidades sociales y solución de problemas.

Concretamente, los trastornos de ansiedad se han vinculado a un mayor riesgo de suicidio si se acompañan de algún trastorno depresivo, pues parecen conformar un puente entre la ideación suicida y el intento de suicidio $(30,31)$. El consumo de sustancias, incluyendo el alcohol, está estrechamente relacionado con el suicidio en todos los grupos de edad a partir de la adolescencia (32-34), razón por la cual la prevención del suicidio debe incluir programas de prevención de adicciones y rehabilitación integral.

Pese a que es innegable la influencia de los trastornos mentales en la conducta suicida, se ha enfatizado que no todas las personas que presentan ideación suicida, intento de suicidio, o incluso que han muerto por daño autoinfligido, tienen algún trastorno mental y/o de la personalidad (35). Investigaciones recientes señalan que el suicidio es más que un problema de salud mental, pues las motivaciones para llevarlo a cabo están vinculadas también a factores sociales y a crisis de vida provocadas por la disminución en el ajuste social, la afectación en las relaciones interpersonales o de pareja, el desempleo y el estrés laboral o financiero $(36,37)$.

\section{Suicidio y Covid-19}

La instauración del Covid-19 trajo consigo muchos más desafíos que la enfermedad misma. La pandemia ha tenido implicaciones tan graves como la muerte de casi un millón de personas en el mundo (38), el sufrimiento por la pérdida de un ser querido con las complicaciones concomitantes en la elaboración del duelo, la crisis económica y el desempleo, las enfermedades mentales que han surgido y que se han exacerbado con el confinamiento en todos los grupos de edad; por el acceso a los medios para provocarse la muerte (39), que aún no se han restringido a pesar de las recomen- 
daciones de la Organización Mundial de la Salud e, incluso, por la polaridad en las manifestaciones afectivas hacia el personal sanitario.

La inminente repercusión de los factores asociados al Covid-19 con el suicidio ha generado la alerta de especialistas alrededor del mundo, quienes advierten del probable incremento en la conducta suicida durante la crisis sanitaria (39-45) e, incluso, una vez que ésta haya sido controlada, pues es un hecho que, a pesar de las predicciones que se puedan generar sobre la «oleada» ${ }^{4}$ de trastornos mentales y suicidios causados por el confinamiento, poco sabemos del impacto real que tendrá en las vidas de las personas.

No obstante que aún es necesaria mayor investigación al respecto, las cifras hasta el momento indican un incremento en la conducta suicida desde diciembre de 2019 y enero de 2020. En México se han reportado suicidios vinculados con la pandemia provocada por Covid-19 y, particularmente, con el confinamiento y sus elementos asociados. Durante los más de 150 días de distanciamiento social en nuestro país, se ha identificado un mayor número de personas (un porcentaje significativo de hombres y adultos jóvenes) (46) con gestos suicidas, en los cuales afortunadamente se ha logrado intervenir. En algunos casos, la actuación ha sido en tiempo real, pero en otros se ha podido llevar a cabo mediante primeros auxilios psicológicos e intervención en crisis vía telefónica o por medio de whatsapp, proporcionados por trabajadores de dependencias gubernamentales o voluntarios de instituciones clínicas y educativas. Se ha observado un incremento en las llamadas solicitando ayuda psicológica conforme los meses han avanzado. De esta forma, en julio de 2020 se ha recibido un mayor número de llamadas que en marzo del mismo año (47). Las solicitudes de apoyo psicológico, pedidas directamente por la persona que lo requiere, o bien por alguien cercano -familiar o amigo- son por sentimientos de soledad y aislamiento, cambios en el estado de ánimo, irritabilidad, tristeza, estrés, miedo, ansiedad, depresión, trastornos del sueño, adicciones, episodios de violencia intrafamiliar, ruptura de relacio- 
nes de pareja, daño en las relaciones interpersonales, ideación suicida e intento de suicidio (48-51).

Con estos datos, se podría afirmar que el Covid-19 ha tenido un impacto significativo en la salud mental y, específicamente, en la muerte autoprovocada; sin embargo, vale la pena ahondar en cada uno de los factores que pudieran estar involucrados en esta asociación.

Uno de los principales radica en la crisis económica y laboral mundial, que está estimada en la pérdida de casi 25 millones de empleos, según la Organización Internacional del Trabajo. De cumplirse estos pronósticos, la pobreza laboral, prevista originalmente para el 2020 en 14 millones en todo el mundo, se estaría situando entre 8.8 y 35 millones de personas (52). América Latina ha tenido un mayor perjuicio que otros países del continente americano, Europa y Asia, con una pérdida de $18.3 \%$ de horas laborales registradas en el segundo trimestre del 2020 , un $14 \%$ por arriba del descenso a nivel mundial en el mismo; Chile, Brasil, Colombia y México son los países más afectados (53).

En nuestro país, se han contabilizado 11.9 millones de personas con suspensión temporal del trabajo sin pago, de los cuales sólo el $42.3 \%$ considera que podría regresar a laborar una vez terminada la contingencia sanitaria (53). De acuerdo con el Instituto Nacional de Estadística y Geografía (INEGI), en cerca del 30\% de los hogares un integrante de la familia perdió su trabajo como consecuencia de la pandemia, y en $65.1 \%$ de las viviendas los ingresos han disminuido (53-57). Las cifras indican que las más afectadas por el desempleo son las mujeres que trabajan en el sector informal (58), incluyendo a las trabajadoras del hogar, que continúan siendo vulnerables a pesar de que, por ley, se les debe incorporar en el Seguro Social.

La crisis económica y laboral, sin duda, conlleva alteración en el autoconcepto y autoestima, estrés exacerbado, desestabilización emocional y sentimientos de vergüenza; culpa, frustración y pérdida que están asociados con ideas sobre la muerte y gestos suicidas. En las crisis económicas más recrudecidas a nivel mundial se ha re- 
gistrado un mayor número de suicidios en ambos sexos, pero mayormente en hombres (59-62).

El confinamiento, por otro lado, se vive de diferente manera dependiendo de las variables que lo acompañan; por ejemplo, la estabilidad laboral y económica, el tipo de vivienda con el que se cuente, el número de miembros de la familia con los que se habita, las relaciones interpersonales que se tengan, y la salud física y mental que se posea.

En algunos casos, el confinamiento se experimenta como distanciamiento social, pero en otros como aislamiento social; es en este último rubro donde las implicaciones emocionales y psicológicas son mayores y, sin embargo, en todos los casos observamos una mayor tendencia a interactuar menos con familiares y amigos -lo cual a veces se percibe como una disminución en la red de apoyo social-; un cambio en la rutina y en los hábitos alimenticios y de sueño; mayor cansancio físico y emocional -que aumenta si se es responsable de un adulto mayor, un menor de edad o alguien con mayor riesgo a presentar complicaciones por Covid-19- y una sensación de sobrecarga en aquellos que trabajan a distancia, pues se ha identificado que el $70 \%$ de las personas trabaja más haciendo bome office, con un promedio de entre 9 y 12 horas laborables por día (63).

El confinamiento incrementa el riesgo de tener problemas interpersonales, de presentar cambios en el estado de ánimo, de aumentar el consumo de alcohol y sustancias ilegales, y de vivir o ser testigo de violencia intrafamiliar ${ }^{5} \mathrm{y}$ abuso sexual -sobre todo hacia las mujeres y niños- (64). El aumento de estresores rebasa los mecanismos de defensa y estilos de afrontamiento que se empleaban habitualmente, lo que exacerba la sintomatología de aquellos que ya presentaban trastornos mentales ${ }^{6} \mathrm{o}$ incluso genera cuadros de depresión, ansiedad, trastorno de estrés agudo y trastorno de estrés postraumático $(64,65,68)$, los cuales, aunados a un sentimiento de desesperanza y a la dificultad para proyectarse en el futuro, conducen a ideación o intento de suicidio. 
La población más vulnerable es la conformada por los adultos mayores y los jóvenes (70). Los adultos mayores que no viven con sus hijos o con algún familiar han tenido que experimentar el confinamiento en soledad, en muchos casos aislados porque, al no saber utilizar satisfactoriamente las herramientas tecnológicas, no se pueden comunicar por este medio con sus hijos o nietos. En el caso de los jóvenes, éstos se enfrentan a altos niveles de tensión por situaciones relacionadas con sus estudios; por ejemplo, la pérdida de la rutina y las relaciones sociales, el cierre de las escuelas, preocupaciones sobre si contarán con los materiales necesarios para tomar clases en línea o, inclusive, si podrán continuar sus estudios o éstos se verán interrumpidos dada la situación económica de sus padres, además de la ansiedad que pueden experimentar por la salud de sus familiares $(64,70,71)$.

Los niños también se han visto mermados en su salud emocional, manifestando cambios conductuales significativos, ansiedad o nerviosismo, irritabilidad expresada en berrinches o rabietas, agitación o soledad. Para ellos tampoco ha sido fácil tener que tomar clases en línea, alterar su rutina, dejar de ver a sus compañeros de escuela y convivir horas extra con su familia. La situación se torna más compleja si el niño tiene alguna discapacidad, vive en hacinamiento o se encuentra en condiciones de mayor vulnerabilidad social, como sería vivir o trabajar en la calle (64). Otro grupo que ha presentado afectaciones importantes en la salud mental es el de aquellos que se encuentran en entornos humanitarios o de conflicto, pues además de que los trastornos mentales son más frecuentes en esta población (64), con la crisis sanitaria, el estrés se ha exacerbado y la posibilidad de atención médica y psicológica ha disminuido.

El duelo es un elemento que ha cambiado durante la crisis sanitaria. Elaborar la pérdida de un ser querido en condiciones ordinarias es suficientemente doloroso y complejo, pero hacerlo durante la pandemia, lo es aún más. La muerte de una persona cercana en tiempos de Covid-19 impide la realización de los ritos funerarios que permiten la asimilación de la pérdida. No poder organizar un 
velorio comunitario ni llevar a niños en edad suficiente a los ritos funerarios, o no mostrar las condolencias de manera presencial y con demostraciones físicas, ha constituido un reto que, en muchas ocasiones, ha devenido en un duelo patológico.

La situación es aún más ininteligible cuando la persona cercana ha fallecido por Covid-19, pues en la mayoría de las ocasiones no pudo darse una despedida física (en el mejor de los casos puede haber una despedida a través de teléfono o videollamada); la enfermedad y la muerte sucedieron demasiado rápido, tanto que no se pudieron procesar emocionalmente; se tiene la sensación de que se dejó al enfermo en un estado estable y no con la salud tan mermada como para concluir en la muerte (72), y se experimenta la sensación de que se pudo haber hecho algo más. Esto conduce generalmente a la aparición de sentimientos de culpa y a estados de depresión y ansiedad.

En diversos países del mundo y en nuestro país se han difundido notas de pacientes que han dado positivos a Covid-19 o que presentaban sospecha de ser portadores del virus, y que tuvieron algún gesto suicida (intento de suicidio que logró contenerse por personal de seguridad, o suicidio generalmente ocurrido al aventarse de la azotea de las instituciones hospitalarias). Los reportes indican que estos pacientes presentaban cuadros de depresión previos, o bien, que no tenían alteraciones emocionales antes del diagnóstico, pero que en el momento en que supieron que podrían tener Covid-19 manifestaron crisis de ansiedad.

Es común que los enfermos por este virus sientan temor e incertidumbre intensos por no saber cómo evolucionarán y si morirán como consecuencia de las complicaciones de la enfermedad. En otros casos, se añade un sentimiento de culpa por la probabilidad de haber contagiado a alguien más, e incluso trauma si es que estuvieron cercanos a una persona que murió por Covid-19 o, aunque éste no sea el caso, si han tenido sobreexposición a noticias relacionadas con el tema. ${ }^{7}$ 
El aislamiento requerido por la enfermedad tiende a incrementar la ansiedad en los pacientes, por lo que es importante que, en la medida de lo posible, estén comunicados con sus familiares, ya sea por vía telefónica o videollamada. En el caso de los menores de edad, la Organización Mundial de la Salud, la Carta Europea de los Derechos de los Niños y el Grupo Mexicano de Psicología Pediátrica, entre otros organismos, señalan que es indispensable que los niños hospitalizados estén acompañados por sus padres, pues una medida inversa contravendría el bienestar integral del paciente (73).

\section{Suicidio y Covid-19 en el personal sanitario}

La profesión de los médicos ha sido considerada desde hace algunos años como una de las profesiones con mayor riesgo en presentar conducta suicida debido a la sobrecarga de trabajo, a la exigencia laboral, a la tendencia a manifestar síndrome de burn out y a las dificultades para contar con medios adecuados para la realización de las actividades solicitadas (74). Durante la pandemia, la ideación suicida y los intentos de suicidio se han incrementado y, desafortunadamente, también lo han hecho los suicidios consumados. Entre las posibles motivaciones de estos hechos se encuentra la exigencia, que no sólo consiste en jornadas laborales extenuantes, sino que en muchas ocasiones se acompaña de la solicitud de realizar tareas distintas a las propias de los médicos, dada la escasez del personal con que cuentan las instituciones hospitalarias. ${ }^{8}$ Además de a mala alimentación e hidratación y de a poco descanso, el personal sanitario se enfrenta a sentimientos de frustración e impotencia; al temor de contagiar a familiares (en consecuencia, al aislamiento del núcleo familiar) y al trauma de ver enfermar y morir a compañeros. A ello se suma el síndrome de burn out y la fatiga por compasión, que tienden a producir cuadros de depresión, ansiedad, trastorno de estrés agudo y trastorno de estrés postraumático. 
El enfrentamiento diario con la enfermedad y la muerte de los pacientes deja de ser algo habitual y se convierte en una situación abrumadora, en particular cuando prevalecen síntomas de indefensión por no contar con los insumos suficientes para el tratamiento adecuado de los pacientes, y tampoco con el equipo de protección personal (76). De acuerdo con reportes recientes, 97,632 trabajadores de la salud se han contagiado de Covid-19 (42\% de los contagios se han dado en enfermeros, $27 \%$ en médicos y $31 \%$ en otros trabajadores como asistentes, técnicos y personal de limpieza). En lo referente a fallecimientos, México registra el mayor índice de letalidad por coronavirus entre los trabajadores de la salud (77). Adicionalmente, el personal sanitario de nuestro país ha tenido que enfrentarse a actos discriminatorios y de violencia verbal, física y psicológica. A diferencia de lo que ha ocurrido en otros países, en los que los trabajadores de la salud son valorados y se les agradece por la labor que hacen diariamente, en México un sector de la población los discrimina y violenta por el aparente temor de que puedan ser transmisores de coronavirus.

A pesar del estigma social que aún existe sobre la enfermedad mental y la solicitud de atención psiquiátrica y psicológica, una gran parte de los trabajadores de la salud se han acercado a las instituciones que ofrecen servicios especializados en salud mental (79, 80), para poder atender las afecciones psicológicas que presentan, lo que evita la cronicidad de los trastornos mentales y abona a la prevención de la conducta suicida.

La prevención del suicidio es para la Organización Mundial de la Salud un imperativo categórico para todos los países (35). Este organismo ha sido enfático en el llamado a las naciones para el diseño de estrategias que favorezcan la salud mental, limiten el acceso a los medios de suicidio, disminuyan los factores de riesgo para la conducta suicida y promuevan los factores de protección de la misma. En la presente crisis sanitaria, tanto la Organización de Naciones Unidas como la Organización Mundial de la Salud y la Organización Panamericana de la Salud $(81,82)$ han distinguido el 
rol tan relevante que tienen los gobiernos de los países en la mitigación de los efectos adversos de la pandemia en todos los sectores de la población.

\section{Bioética y suicidio}

El suicidio está lejos de dejar de ser una problemática mundial, pues la inserción del individuo en la sociedad es indiscutible; quien ha acabado con su vida rompe el tejido social y deja una huella imborrable. Podemos suponer que el sufrimiento de aquel que presenta algún gesto suicida es elevado, y que seguramente atraviesa por problemáticas emocionales y crisis de vida que le suponen un cuestionamiento sobre el sentido de su existencia. Reconocer a estos dolientes como personas con dignidad intrínseca e inalienable es fundamental, pero lo es también atender su salud física y mental.

La enfermedad y la muerte nos recuerdan la fragilidad propia del ser humano, pero en muchos casos también la vulnerabilidad social a la que se enfrenta un grupo poblacional significativo por la pobreza en la que se encuentran, o bien, por el acceso limitado a los servicios sanitarios. En esta área, la de la salud integral, a nivel mundial, pero sobre todo en América Latina, y en particular en México, existen deficiencias relevantes y desacato al principio de justicia distributiva, pues son muchos los grupos vulnerables que no tienen acceso a una atención de calidad para proteger su salud.

Este es el caso de las personas que padecen alguna enfermedad mental y que carecen de recursos económicos suficientes para asistir a una consulta psiquiátrica o psicológica privada, que no acceden a instituciones de atención pública de especialidad, o que tienen que abandonar su tratamiento farmacológico por no tener dinero para pagarlo. Las fallas en la justicia distributiva también hacen referencia a los recursos humanos, pues los médicos especialistas en salud mental en nuestro país se concentran en Ciudad de México, 
Monterrey y Guadalajara, por lo que las personas que no se pueden trasladar a esas ciudades tienen menor probabilidad de ser atendidas por un especialista. La falta de atención en salud mental no afecta exclusivamente al área de salud, sino también a las áreas académica, laboral y social.

Pese a esta imperiosa necesidad por atender la salud mental, los recursos asignados anualmente a este sector han sufrido un recorte presupuestal en promedio del $2.7 \%$ en el mundo (64). En nuestro país, el porcentaje presupuestal de inversión pública en salud mental se encuentra muy por debajo del promedio de los países desarrollados e, incluso, de otras naciones en América Latina.

Las implicaciones que esto conlleva en la vida social y comunitaria son elevadas. Desde esta perspectiva, la responsabilidad de brindar apoyo a los que más lo necesitan no es exclusivamente de los programas gubernamentales, sino también de los miembros de la sociedad en sí mismos, apegándose a los principios de sociabilidad y subsidiariedad. Desde la sociabilidad, todos los miembros de una comunidad se comprometen a colaborar en la obtención del bien de todos; una persona se realiza a sí misma cuando favorece la realización del bien de los otros. Desde la subsidiariedad, la sociedad tiene la responsabilidad de identificar qué elementos propios son promotores del suicidio de uno de sus miembros y qué acciones son las que se deben implementar para prevenir este tipo de conductas (84); por ejemplo, en la disminución del estigma social, en el apoyo a quienes están atravesando por dificultades importantes durante el confinamiento; en quienes manifiestan crisis emocionales o en quienes arriesgan su vida por el bienestar de la población, como el personal sanitario.

Una persona con conducta suicida vive en soledad y aislamiento el proceso emocional por el que atraviesa. Al encontrarse afectivamente en un estado de embotamiento y con pocas posibilidades de identificar de manera efectiva distintas soluciones a sus problemáticas, considera que la única alternativa que tiene es morir. Es en 
esos momentos cuando necesita una red de apoyo social; un grupo de personas que, desde la solidaridad, comprendan su sufrimiento; que desde la sociabilidad promuevan su bienestar, y que desde la subsidiariedad reconozcan que es una persona que requiere atención psicológica pero que, muy probablemente, no se encuentre en condiciones para buscarla por sí mismo.

\section{Conclusiones}

El suicidio es una problemática de salud pública que se ha incrementado de manera alarmante en México y en el mundo. La pandemia del Covid-19 presenta desafíos aún mayores que los de la propia enfermedad. El confinamiento ha tenido un gran impacto en las vidas de las personas, en términos sanitarios, económicos, laborales y sociales. Específicamente en el área de la salud mental, se están comenzando a ver los estragos del mismo; sin embargo, aún es pronto para identificar con claridad el impacto que se tendrá en el corto, mediano y largo plazo en el desarrollo de trastornos mentales, crisis de vida y conducta suicida.

El tratamiento para el suicidio es multimodal. Las maniobras de intervención deben abocarse a la atención efectiva de los trastornos mentales; a la reducción del estigma social; a la implementación de programas preventivos y a la creación de prácticas para la reducción del consumo de alcohol y estupefacientes; a la restricción del acceso a los medios de suicidio; a la resolución de problemáticas severas y a la disminución de los marcadores de desventaja social.

Resulta cardinal dar seguimiento a las enfermedades mentales, crisis de vida, ideación e intento de suicidio que se están presentando en diferentes sectores de la población a causa del Covid-19. No obstante, es trascendental la promoción del sentido de comunidad y la relevancia del bien común, pues sólo con el respeto a la digni- 
dad de la persona y con la aplicación de los principios de sociabilidad, subsidiariedad y solidaridad es como se podrá salvaguardar la vida humana.

\section{Notas bibliográficas}

${ }^{1}$ Las entidades federativas con mayor tasa de suicidios en la población de 15 a 29 años son: Guanajuato (15.9), Chihuahua (15.7), Campeche (14.8) y Aguascalientes (14.1).

2 De acuerdo con cifras del Instituto Nacional de Estadística y Geografía, los suicidios en el rango de edad de 10 a 14 años fueron de $7.6 \%$ en mujeres y de $2.7 \%$ en hombres; en el de 15 a 19 años, $21.8 \%$ en mujeres y 15.4 en hombres, y en el de 20 a 24 años, $16.9 \%$ entre mujeres y $15.4 \%$ entre hombres.

${ }^{3}$ La conducta suicida involucra la ideación suicida, que generalmente deviene de las ideas sobre la muerte y que puede ser estructurada o no estructurada; el intento de suicidio, que puede ser de alta o de baja letalidad, y el suicidio o suicidio consumado, como también es conocido. El proceso de la conducta suicida puede ser breve o prolongado, dependiendo de las características particulares de cada persona y su situación de vida; sin embargo, es importante considerar que generalmente en los adolescentes y adultos jóvenes este proceso es corto, de manera que se encuentra un mayor riesgo de presentar intento de suicidio cercano a la ideación suicida antecedente.

${ }^{4}$ Este término se ha usado frecuentemente en los medios de comunicación impresos para describir las probables consecuencias negativas respecto de la salud mental y conducta suicida relacionadas con la pandemia por Covid-19.

${ }^{5}$ Las estimaciones indican que en todo el mundo se pueden producir 31 millones de casos adicionales de violencia de género si continúa el confinamiento durante 6 meses o más (64). En México, la violencia intrafamiliar se ha incrementado el $120 \%$ desde que comenzó la pandemia (66\% violencia física y $22 \%$ violencia emocional) (66), lo cual se ve reflejado en las llamadas de ayuda que se hacen a las líneas de atención telefónica sobre este tema, las cuales observan un incremento notable con respecto al año 2019 (67).

${ }^{6}$ En algunos casos, aquéllos ya diagnosticados con trastornos mentales han abandonado su tratamiento por cuestiones económicas o por el miedo a contagiarse de Covid-19 (cuando tienen que asistir a un consultorio o institución hospitalaria). ${ }^{7}$ La sobreexposición a noticias puede alterar el sueño y la estabilidad emocional de las personas; es por ello que se recomienda consultar las noticias (por televisión, radio o redes sociales) exclusivamente dos veces al día y no inmediatamente antes de dormir.

${ }^{8}$ En México, médicos del Hospital 20 de Noviembre del ISSSTE denunciaron que, por falta de personal de aseo, se les solicitó que limpiaran su área de trabajo una vez concluidas sus labores (75). 


\section{Referencias bibliográficas}

1. Organización Mundial de la Salud. Suicidio. Datos y cifras. 2019. [Consultado el 28 de agosto de 2020]. Recuperado en: https://www.who.int/es/news-room/factsheets/detail/suicide

2. Organización Mundial de la Salud. World Health Statistics data visualizations dashboard. 2019. [Consultado el 28 de agosto de 2020]. Recuperado en: http:// apps. who.int/gho/data/node.sdg.3-4-viz-2?lang=en

3. Organización Mundial de la Salud. Cada 40 segundos se suicida una persona. 2019. [Consultado el 31 de agosto de 2020]. Recuperado en: https://www.who.int/ es/news-room/detail/09-09-2019-suicide-one-person-dies-every-40-seconds

4. Armstrong M. Suicide rate around the world. Statista.com. 2019. [Consultado el 31 de agosto de 2020]. Recuperado en: https://www.statista.com/chart/15 390/global-suicide-rates/

5. Lee P, Roser M, Ortiz-Ospina E. Suicide deaths by age, World. OurWorldIn Data.org. 2018. [Consultado el 31 de agosto de 2020]. Recuperado en: https://our worldindata.org/suicide

6. Instituto Nacional de Estadística y Geografía. Estadísticas a propósito del día mundial para la prevención del suicidio (datos nacionales). 2018. [Consultado el 31 de agosto de 2020]. Recuperado en: https://www.inegi.org.mx/contenidos/saladeprensa/aproposito/2018/suicidios2018_Nal.pdf https://doi.org/10.4060/ca8614es

7. Instituto Nacional de Estadística y Geografía. Estadísticas a propósito del día mundial para la prevención del suicidio (datos nacionales). 2017. [Consultado el 31 de agosto de 2020]. Recuperado en: http://www.inegi.org.mx/saladeprensa/ aproposito/2017/suicidios2017_Nal.pdf https://doi.org/10.4060/ca8614es

8. Sánchez-Cervantes F, Serrano-González RE, Márquez-Caraveo M. Suicidio en menores de 20 años. México 1998-2011. Salud Mental. 2015; 38(5): 379-389. https://doi.org/10.17711/sm.0185-3325.2015.051

9. Ludwig B, Roy B, Wang QZ, Birur B, Dwivedi Y. The life span model of suicide and Its Neurobiological Foundation. Frontiers in Neuroscience. 2017; 11, 74. https:// doi.org/10.3389/fnins.2017.00074

10. Jaiswal SV, Faye AD, Gore SP, Shah HR, Kamath RM. Stressful life events, hopelessness, and suicidal intent in patients admitted with attempted suicide in a tertiary care general hospital. Journal of Postgraduate Medicine. 2016; 62(2): 102104. https://doi.org/10.4103/0022-3859.180556

11. Chau K, Kabuth B, Chau N. Gender and family disparities in suicide attempt and role of socioeconomic, school, and health-related difficulties in early adolescence. Biomed Research International. 2014; 13. https://doi.org/10.1155/2014/ 314521

12. Chau K, Kabuth B, Chau N. Association between suicide ideation and attempts and being an immigrant among adolescents, and the role of socioeconomic factors and school, behavior, and health-related difficulties. International Journal of 
Suicidio: el impacto del Covid-19 en la salud mental

Environmental Research and Public Health. 2016; 13(11): 15. https://doi.org/10.3390 /ijerph13111070

13. Ruiz Rodríguez A, Rivas Gómez A. Disfunción familiar en pacientes con intento de suicidio en el área de urgencias pediátricas. Salud Pública de México. 2017; 59(3): 209-210. https://doi.org/10.21149/8326

14. Albores Gallo L, Sauceda García JM, Ruiz Velasco S, Roque Santiago E. Bullying and its association with psychiatric disorders in a Mexican students sample. Salud Pública de México. 2011; 53(3): 220-227.

15. Bruwer B, Govender R, Bishop M, Williams DR, Stein DJ, Seedat S. Association between childhood adversities and long-term suicidality among South Africans from the results of the South African stress and health study: a cross-sectional study. BMJ Open. 2017; 4(6): 14. https://doi.org/10.1136/bmjopen-2013-004644

16. Khemiri L, Jokinen J, Runeson B, Jayaram-Lindstrom N. Suicide risk associated with experience of violence and impulsivity in alcohol dependent patients. Scientific Reports. 2016; 6(11). https://doi.org/10.1038/srep19373

17. Haglund $A$, Lindh $A U$, Lysell $H$, Renberg ES, Jokinen J, Waern $M$ et al. Interpersonal violence and the prediction of short-term risk of repeat suicide attempt. Scientific Reports. 2016; 6, 8. https://doi.org/10.1038/srep36892

18. Tovilla Zárate CA, González Castro T B, Juárez Rojop I, García SP, Velázquez Sánchez MP, Villar Soto M, et al. Study on genes of the serotonergic system and suicidal behavior: protocol for a case-control study in Mexican population. BMC Psychiatry. 2014; 14: 5. https://doi.org/10.1186/1471-244x-14-29

19. Pandey GN, Rizavi HS, Zhang H, Bhaumik R, Ren XG. The Expression of the suicide-associated gene SKA2 is decreased in the prefrontal cortex of suicide victims but not of nonsuicidal patients. International Journal of Neuropsychopharmacology. 2016; 19(8): 10. https://doi.org/10.1093/ijnp/pyw015

20. Niculescu AB, Levey D, Le-Niculescu H, Niculescu E, Kurian SM, Salomon D. Psychiatric blood biomarkers: avoiding jumping to premature negative or positive conclusions. Mol Psychiatry. 2015; 20(3): 286-288. https://doi.org/10.1038/mp. 2014.180

21. Desphande G, Baxi M, Witte T, Robinson JL. A Neural basis for the acquired capability for suicide. Frontiers in Psychiatry. 2016; 7: 19. https://doi.org/10.3389/ fpsyt.2016.00125

22. Chang BP, Franklin JC, Ribeiro JD, Fox FR, Bentley KH, Kleiman EM, et al. Biological risk factors for suicidal behaviors: a meta-analysis. Translational Psychiatry. 2016; 6: 8. https://doi.org/10.1038/tp.2016.165

23. Breslau J, Gilman SE, Stein BD, Ruder T, Gmelin T, Miller E. Sex differences in recent first-onset depression in an epidemiological sample of adolescents. Translational Psychiatry. 2017; 7: 6. https://doi.org/10.1038/tp.2017.105

24. Ibrahim N, Amit N, \& Suen MW. Psychological Factors as predictors of suicidal ideation among adolescents in Malaysia. Plos One. 2014; 9(10): 6. https://doi.org/ 10.1371/journal.pone. 0110670

Medicina y Ética - Enero-Marzo 2021 - Vol. 32 - Núm. 1 


\section{E. Benítez Camacho}

25. Ibrahim N, Amit N, Suen MWY. Psychological factors as predictors of suicidal ideation among adolescents in Malaysia. Plos One. 2014; 9(10): 6. https://doi.org/ 10.1371/journal.pone. 0110670

26. Zhang S, Chen JM, Kuang L, Cao J, Zhang H, Ai M, Fang WD, et al. Association between abnormal default mode network activity and suicidality in depressed adolescents. BMC Psychiatry. 2016; 16: 10. https://doi.org/10.1186/s12888-016-1047-7 27. Borges G, Benjet C, Medina-Mora ME, Orozco R, Familiar I, Nock MK, et al. Service use among Mexico City adolescents with suicidality. Journal of Affective Disorders. 2010; 120(1-3): 32-39. https://doi.org/10.1016/j.jad.2009.04.008

28. Borges G, Orozco R, Mora ME. Risk index for attempted suicide in Mexico. Salud Pública de México. 2012; 54(6): 595-606. https://doi.org/10.1590/s0036-3634 2012000600008

29. Paruk S, Karim E. Update on adolescent mental health. Samj South African Medical Journal. 2016; 106(6): 23-25. https://doi.org/10.7196/SAMJ.2016.v106i6. 10943

30. Strandheim A, Bjerkeset O, Gunnell D, Bjornelv S, Holmen TL, Bentzen N. Risk factors for suicidal thoughts in adolescence-a prospective cohort study: the Young-HUNT study. BMJ Open. 2014; 4(8): 8. https://doi.org/10.1136/bmjopen-2014005867

31. Baca E, Fuensanta A. Factores de riesgo de la conducta suicida asociados a trastornos depresivos y ansiedad. Salud Mental. 2014; 37(5): 373-380. https://doi. org/10.17711/SM.0185-3325.2014.044

32. Arensman E, Bennardi M, Larkin C, Wall A, McAuliffe C, McCarthy J. Suicide among young people and adults in Ireland: Method, characteristics, toxicological analysis and substance abuse histories compared. Plos One. 2016; 11(11): 14. https://doi.org/10.1371/journal.pone.0166881

33. Borges G, Benjet C, Orozco R, Medina Mora ME, Menéndez D. Alcohol, cannabis and other drugs and subsequent suicide ideation and attempt among young Mexicans. Journal of Psychiatric Research. 2017; 91: 74-82. https://doi.org/10.10 16/j.jpsychires.2017.02.025

34. Ferrari AJ, Norman RE, Freedman G, Baxter AJ, Pirkis JE, Harris, MG, et al. The burden attributable to mental and substance use disorders as risk factors for suicide: Findings from the global burden of disease study 2010. Plos One. 2014; 9(4): 11. https://doi.org/10.1371/journal.pone.0091936

35. Organización Mundial de la Salud. Prevención del suicidio, un imperativo global. 2014. [Consultado el 1 de septiembre de 2020]. Recuperado en: https:// apps.who. int/iris/bitstream/handle/10665/136083/9789275318508_spa.pdf;jses sionidF50 E2767A011FB080F09C842E189E976?sequence $=1$

36. Centros para el Control y la Prevención de Enfermedades. Las tasas de suicidio están aumentando por todos los EE.UU. 2018. [Consultado 1 el 1 de septiembre de 2020]. Recuperado en: https://www.cdc.gov/spanish/mediosdecomunicacion/comu nicados/p_vs_suicidio_060718.html https://doi.org/10.4185/rlcs-20181283 
Suicidio: el impacto del Covid-19 en la salud mental

37. Healy M. Aumentan en más de un $30 \%$ los suicidios en Estados Unidos. Los Ángeles Times. 2018. [Consultado el 1 de septiembre de 2020]. Recuperado en: http://www.latimes.com/espanol/vidayestilo/la-es-aumentan-en-mas-de-un-30-lossuicidios-en-estados-unidos-20180607-story.html https://doi.org/10.4272/978-849745-562-6.ch2 https://doi.org/10.1590/scielopreprints.868

38. El mundo. Mapa del coronavirus: expansión en cifras del Covid-19 en el mundo. 2020. [Consultado el 7 de septiembre de 2020]. Recuperado en: https:// www.elmun do.es/ciencia-y-salud/salud/2020/03/02/5e5cd4ebfc6c83632e8b 4644.html

39. Brown S, Shuman DL. Suicide in the time of Covid-19: A perfect storm. The Journal of Rural Health. 2020; 1-4. https://doi.org/ 10.1111/jrh.12458

40. Thakur V, Jain A. Covid-19-suicides: A global pychological pandemic. Brain Behav Immun. 2020; 88: 952-953. Recuperado en: https://www.ncbi.nlm.nih.gov/ pmc/articles/PMC7177120/ https://doi.org/10.1016/j.bbi.2020.04.062

41. Ribeiro E. Fighting suicide during Covid-19: Lessons from past pandemics and recessions. VOX EU. 2020. [Consultado el 8 de septiembre de 2020]. Recuperado en: https://voxeu.org/article/fighting-suicide-during-Covid-19

42. Sher L. The impact of the Covid-19 pandemic on suicide rates. QJM: An International Journal of Medicine; hcaa202. https://doi.org/ 10.1093/qjmed/hcaa202

43. Carey B. Is the pandemic sparking suicide? The New York Times. 2020. [Consultado el 8 de septiembre de 2020]. Recuperado en: https://www.nytimes.com / 2020/05/19/health/pandemic-coronavirus-suicide-health.html

44. Bach T. Will suicides rise because oof Covid-19? [Internet] US News, 2020. [Consultado el 8 de septiembre de 2020]. Recuperado en: https://www.usnews. com/news/healthiest-communities/articles/2020-05-22/experts-warn-of-a-surge-ofsuicides-tied-to-the-coronavirus-pandemic

45. Reger MA, Stanley IH, Joiner TE. Suicide mortality and coronavirus disease. JAMA Psychiatry. 2020. https://doi.org/10.1001/jamapsychiatry.2020.1060

46. Infobae. Creció el número de suicidios entre jóvenes mexicanos durante la emergencia sanitaria por Covid-19. INFOBAE. 2020. [Consultado el 7 de septiembre de 2020]. Recuperado en: https://www.infobae.com/america/mexico/2020/08/ 15/crecio-el-numero-de-suicidios-entre-jovenes-mexicanos-durante-la-emergencia-sanitaria-por-Covid-19/ https://doi.org/10.20318/recs.2020.5441

47. Excelsior. Suicidio durante la pandemia por Covid-19. 2020. [Consultado el 2 de septiembre de 2020]. Recuperado en: https://www.excelsior.com.mx/nacional/ suicidio-durante-la-pandemia-por-Covid-19/1397483 https://doi.org/10.22592/ ode20 20nespa4

48. Mejía B. Las llamadas a líneas directas de suicidio están aumentando por el coronavirus. Los consejeros sienten el dolor que está causando. Los Ángeles Times. 2020. [Consultado el 8 de septiembre de 2020]. Recuperado en:https:// www.lati mes.com/espanol/california/articulo/2020-04-05/las-Ilamadas-a-las-lineas-directas-de-suicidio-estan-aumentando-por-el-coronavirus-los-consejerossienten-el-dolor-que-esta-causando https://doi.org/10.1787/888934073626

Medicina y Ética - Enero-Marzo 2021 - Vol. 32 - Núm. 1 
49. INFOBAE. Apoyo psicológico y asesoría jurídica por violencia familiar, servicios que más solicitan en call centers de ayuda ante Covid-19. 2020. [Consultado el 8 de septiembre de 2020]. Recuperado en: https://www.infobae.com/america/mexico/2020/04/27/apoyo-psicologico-y-asesoria-juridica-por-violencia-familiar-servicios-que-mas-solicitan-en-call-centers-de-ayuda-ante-Covid-19 https://doi.org/ 10.1016/j.psiq.2020.08.001

50. Miranda F. CdMx activa línea telefónica para dar consulta psicológica por coronavirus. Milenio. 2020. [Consultado el 8 de septiembre de 2020]. Recuperado en: https://www.milenio.com/politica/comunidad/coronavirus-cdmx-activa-linea-telefonica-consulta-psicológica.

51. Instituto Mexicano del Seguro Social. Por la emergencia sanitaria Covid-19 el IMSS ha atendido 1379 llamadas para la atención en salud mental. 2020. [Consultado el 8 de septiembre de 2020]. Recuperado en: http://www.imss.gob.mx/prensa/archivo/202006/394 https://doi.org/10.1787/9789264288300-12-es

52. AFP. Coronavirus destruiría 25 millones de empleos en el mundo. Empresas \& Management. 2020. [Consultado el 7 de septiembre de 2020]. Recuperado en: https://www.estrategiaynegocios.net/lasclavesdeldia/1365035-330/coronavirusdestruir\%C3\%ADa-25-millones-de-empleos-en-el-mundo https://doi.org/10.30875/ 08b30766-es

53. Archiniegas Y. OIT: América Latina es la región con más empleos perdidos por cuenta de la pandemia. France 24. 2020. [Consultado el 7 de septiembre de 2020]. Recuperado en: https://www.france24.com/es/20200701-oit-latinoamerica-desempleo-economia-pandemia-Covid19 https://doi.org/10.18356/35991e4a-es 54. LatinUs. Suman 15.7 millones de desempleados en México por Covid-19: INEGI. 2020. [Consultado el 2 de septiembre de 2020]. Recuperado en: https://latinus. us/2020/07/23/suman-15-7-millones-desempleados-mexico-Covid19-inegi/ 55. EFE. El desempleo desafía la recuperación en ' $V$ ' que espera López Obrador. Expansión. 2020. Recuperado en: https://expansion.mx/economia/2020/06/13/des empleo-desafia-recuperacion-que-espera-amlo https://doi.org/10.32468/be.494 56. González LM. 1 millón... 12 millones, ¿cuántos empleos se han perdido por el Covid-19? López-Dóriga Digital. 2020. Recuperado en: https://lopez doriga.com/ opinion/1-millon-12-millones-cuantos-empleos-se-han-perdido-por-el-Covid-19/

57. López-Dóriga J. El impacto de la pandemia en el empleo. El Economista. 2020. Recuperado en: https://www.eleconomista.com.mx/opinion/El-impacto-de-la-pandemia-en-el-empleo-20200603-0105.html https://doi.org/10.2307/j.ctv6hp3sq.15

58. El Heraldo de México. Mujeres, las más afectadas por el desempleo durante pandemia por Covid-19. 2020. [Consultado el 2 de septiembre de 2020]. Recuperado en: https://heraldodemexico.com.mx/pais/desempleo-Covid-19-mujeres-pandemia-pobreza-mexico/ https://doi.org/10.22592/ode2020nespa4

59. Iglesias GC, Sáiz PA, Burón P, Sánchez Lasheras F, Jiménez Treviño L, Fernández Artamendi $\mathrm{S}$, et. al. Suicidio, desempleo y recesión económica en España. Revista de Psiquiatría y Salud Mental, 2017; 1(2), 70-77. https://doi.org/10.1016/ j.rpsm.2016.04.005 
Suicidio: el impacto del Covid-19 en la salud mental

60. Oyesana M, López-Moringo J, Dutta R. Systematic review of suicide in economic recession. World J Psychiatry. 2015; 5(2): 243-254. https://doi.org/10.5498/wj p.v5.i2. 243

61. Fernández A. Prevención del suicidio durante una crisis económica. 2016. [Consultado el 7 de septiembre de 2020]. Recuperado en: https://www.asistencia alsui cida.org.ar/post/prevención-del-suicidio-durante-una-crisis-económica https:/ /doi.org/10.2307/j.ctt1zgwm3k.7

62. Celada FJ, Quiroga A, Mohedano A, Aliaga I, Fernández C, Martin JL. Tentativa suicida atendida por los servicios de urgencias de Castilla-La Mancha y crisis económica. Revista Científica de la Sociedad Española de Medicina de Urgencias y Emergencias. 2018; 30(4). https://doi.org/10.1016/j.oftal.2020.05.001

63. Hernández G. Tiempos de Covid-19, el $70 \%$ de las personas trabaja más haciendo home office. Factor Capital Humano, El Economista. 2020. Recuperado en: https://factorcapitalhumano.com/mundo-del-trabajo/tiempos-de-Covid-19-el70-de-las-personas-trabaja-mas-haciendo-home-office/2020/05/ https://doi.org/ 10.12795/e-rips.2020.i01.06

64. Organización de Naciones Unidas. Informe de políticas: Covid-19 y la necesidad de actuar en relación con la salud mental. 2020. [Consultado el 7 de septiembre de 2020]. Recuperado en: https://www.un.org/es/coronavirus/articles/servicios-de-salud-mental-respuesta-gobiernos-Covid-19 https://doi.org/10.18356/ 7cc92199-es

65. Riesgo M. El confinamiento prolongado puede aumentar el riesgo de suicidio. Gaceta Médica, 2020. [Consultado el 7 de septiembre de 2020]. Recuperado en: https://gacetamedica.com/investigacion/el-confinamiento-prolongado-puede-aumentar-el-riesgo-de-suicidio/ https://doi.org/10.1016/s1134-2072(10)70290-5

66. Monroy J. SEGOB: violencia intrafamiliar aumentó $120 \%$ desde la emergencia del Covid-19. El Economista, 2020. [Consultado el 7 de septiembre de 2020]. Recuperado en: https://www.eleconomista.com.mx/politica/Segob-violencia-intrafamiliar-aumento-120-desde-la-emergencia-del-Covid-19-20200416-0111.html https:// doi.org /10.33210/ca.v9i2.316

67. Ramírez M, Ramírez de Garay. Auxilio apremiante: el aumento de las llamadas por violencia de género en la CdMx. México Evalúa. 2020. [Consultado el 7 de septiembre de 2020]. Recuperado en: https://www.mexicoevalua.org/auxilioapremiante-el-aumento-de-las-Ilamadas-por-violencia-de-genero-en-la-cdmx/ https://doi.org/10.2307/j.ctv103xb4t.12

68. Clay RA. Covid-19 and suicide. How the pandemic will affect suicide rates is still unknown, but there's much psychologists can do to mitigate its impact. American Psychological Association. [Consultado el 2 de septiembre de 2020]. 2020; 51(4). Recuperado en: https://www.apa.org/monitor/2020/06/Covid-suicide 69. Notimex. Medidas de confinamiento por Covid-19 aumentarán índices de depresión y suicidio, estima la OMS. El Financiero. 2020. [Consultado el 7 de septiembre de 2020].

Medicina y Ética - Enero-Marzo 2021 - Vol. 32 - Núm. 1 
70. Ordaz A. Jóvenes y adultos mayores, los más vulnerables al suicidio en pandemia, alertan. Forbes. 2020. [Consultado el 7 de septiembre de 2020]. Recuperado en: https://www.forbes.com.mx/noticias-jovenes-adultos-mayores-vulnerables-suicidio-pandemia/ https://doi.org/10.2307/j.ctt1zgwm3k.5

71. Ciechalski S, Walters S, Kaufman S. College students brace for the «second curve» of Covid-19-its mental health impact. NBC News. 2020. [Consultado el 2 de septiembre de 2020]. Recuperado en: https://www.nbcnews.com/news/us-news/ college-students-brace-second-curve-Covid-19-its-mental-health-n1238740 https:/ /doi.org/10.1126/science.abc4071

72. Gallo M, Llaca C, Adame MJ. Duelo por Covid-19. Tooltoys. 2020. [Consultado el 7 de septiembre de 2020]. Recuperado en: https://cuidadospaliativos.org/blog/ wp-content/uploads/2020/04/Duelo-por-Covid-19.pdf https://doi.org/10.22592/ ode20 20nespa4

73. Busqueta MF. Los padres deben acompañar a sus hijos hospitalizados siempre, incluso por CoviD-19. Fundación Vuela. 2020. [Consultado el 8 de septiembre de 2020]. Recuperado en: https://vuela.org/2020/07/06/los-padres-deben-acompanar-a-sus-hijos-hospitalizados-siempre-incluso-por-Covid-19/ https://doi.org/ 10.36576/summa.7787

74. Cano Langreo M, Cicirello Salas S, López López A, Aguilar Vela M. Marco actual del suicidio e ideas suicidas en personal sanitario. Med. Segur. Trab. 2014; 60(234): 198-218. https://doi.org/10.4321/S0465-546X2014000100015 https://doi. org/10.4321/s0465-546x2014000100015

75. Redacción de Animal Político. Por falta de personal de aseo piden a empleados de hospital Covid del ISSSTE limpiar su área de trabajo. Animal Político. 2020. [Consultado el 9 de septiembre de 2020]. Recuperado en: https://www.ani malpolitico. com/2020/06/personal-aseo-empleados-hospital-issste-limpiar-area-de-trabajo/ https://doi.org/10.36576/summa.14111

76. Vera R. Tragedia en el sector salud: "Morimos en medio de la indiferencia». Proceso. 2020. [Consultado el 9 de septiembre de 2020]. Recuperado en: https:// www. proceso.com.mx/634084/coronavirus-en-mexico-tragedia-en-el-sector-salud-morimos-en-medio-de-la-indiferencia https://doi.org/10. 2307/j.ctv16zjhg3.6

77. Associated Press. México lidera en trabajadores de la salud muertos por Covid-19. Chicago Tribune. 2020. [Consultado el 9 de septiembre de 2020]. Recuperado en: https://www.chicagotribune.com/espanol/sns-es-coronavirus-mexico-lidera-trabajadores-de-salud-muertos-Covid-20200903-hfmergrh3nf6bbh3k7nfgind pq-story.html https://doi.org/10.4060/ca8560es

78. Guzmán S. CONAPRED registra 213 quejas por actos de discriminación por Covid-19. El Financiero. 2020. [Consultado el 9 de septiembre de 2020]. Recuperado en: https://www.elfinanciero.com.mx/nacional/conapred-registra-213-quejaspor-actos-de-discriminacion-por-Covid-19 https://doi.org/10.22592/ode2020nespa4 79. Noticias ONU. Ante un posible aumento de los suicidios por el coronavirus, la ONU pide tomar medidas para cuidar la salud mental. Organización de Naciones 
Suicidio: el impacto del Covid-19 en la salud mental

Unidas, 2020. [Consultado el 9 de septiembre de 2020]. Recuperado en: https:// news.un.org/es/story/2020/05/1474312 https://doi.org/10.2307/j.ctt22p7 gf8.5

80. Centros de Integración Juvenil. Covid-19: impacto de la pandemia y atención integral del suicidio. Cuidar al que cuida. Gobierno de México. 2020. [Consultado el 9 de septiembre de 2020]. Recuperado en: https://www.gob.mx/salud/cij/es/articulos/Covid-19-impacto-de-la-pandemia-y-atencion-integral-de-la-salud-cuidar-alque-cuida?idiom=es https://doi.org/10.1016/j.enfcli.2020.05.006

81. Organización Panamericana de la Salud. Seminario web: prevención del suicidio en tiempos de Covid-19. 2020. [Consultado el 9 de septiembre de 2020]. Recuperado en: https://www.paho.org/es/eventos/seminario-web-prevencion-suicidio-tiempos-Covid-19 https://doi.org/10.4060/ca8979es

82. Guterres A. Los servicios de salud mental son parte esencial de todas las respuestas de los gobiernos ante el Covid-19. Organización de Naciones Unidas. 2020. [Consultado el 9 de septiembre de 2020]. Recuperado en: https://www.un. org/es/coronavirus/articles/servicios-de-salud-mental-respuesta-gobiernos-Covid$19 \mathrm{https}: / /$ doi.org/10.35247/buengob_29_05

83. Katz G, Lazcano E, Madrigal E. La salud mental en el ámbito poblacional: la utopía de la psiquiatría social en países de bajos ingresos. Salud Pública de México. 2017; 59(4): 351-353. https://doi.org/10.21149/8860

84. Sgreccia E. Manual de Bioética I. Fundamentos y ética biomédica. Madrid: Biblioteca de Autores Cristianos, 2009. 
